\title{
Niveau D'etudes, Style Educatif Des Parents Et Resilience Scolaire Chez Des Enfants Deficients Auditifs De L'ecole Ivoirienne Pour Les Sourds (ECIS)
}

\author{
Kouassi Affoué Mélissa \\ Doctorante, Université Félix Houphouët-Boigny / Côte d’Ivoire \\ UFR / Sciences de l'Homme et de la Société \\ Laboratoire de Psychologie Génétique Différentielle
}

doi: 10.19044/esj.2016.v12n28p219 URL:http://dx.doi.org/10.19044/esj.2016.v12n28p219

\begin{abstract}
The present work analyzes from the perception of disability, which means that it is an inability to be gifted in some areas, including the school environment. In this perspective, it analyses the operation of parent and disabled child relationship. Carters of the relationship between the level of education, the educational style of parents and school resilience in hearing impaired children, of Ivorian school of deaf. To this end, we were interested in 18 ivoirians children and their parents, 9 girls and 9 boys aged 10 to 12 years, from disadvantaged social background. From the scale of parental educational styles of Terrisse and Larose (2000), the inventory forms of resilience of Békaert, Masclet and Caron (2011) and the Rorschach test (1921), we observed the different educational styles and types of resilient behavior. Overall, the results obtained show that, the educational level and educational style parents, influence the different forms of academic resilience, in hearing deficient children. However, this work could not demonstrate the relationship of the level of secondary school and academic resilience challenge type. The results validate the whole analysis of certain theoretical constructs and the facts noted by other studies, according to which, parents play a role in the autonomy and self-determination of disabled children.
\end{abstract}

Keywords: Level studies of parents- parental educational style- school resilience- hearing impairment- disability

\section{Introduction}

Le développement humain est une succession d'étapes qui marque la vie d'un individu. Bien qu'il existe des lois dans l’évolution de l'espèce, telle 
que par l'exemple l'acquisition de la marche bipède vers l'âge de 12 mois, les trajectoires développementales sont différentes d'un individu à un autre. Les chercheurs ont évoqués pour expliquer ces différences, les milieux dans lesquels nous évoluons. En effet, ce n’est que sous l'influence du milieu dans lequel il vit, et en vertu de ce que le contact avec son entourage apporte, qu'un être humain devient un individu d'un type déterminé (Sillamy, 2003). Ainsi, le cadre de vie, les croyances culturelles, l'ethnie, les appartenances religieuses, la collectivité ou le réseau social et les membres de la famille sont autant de facteurs présents dans l'environnement proche d'un être humain qui l'influencent. Les travaux d'Anthony (1974) ont par ailleurs, insisté sur l'influence du milieu familial, quant au développement de l'individu.

La famille constitue donc, la niche développementale. En d'autres termes, elle a pour rôle d'orienter, d'aider les individus à comprendre et à assimiler les structures culturelles complexes auxquelles ils doivent s'adapter, dans une société donnée. L’adaptation nécessite certaines capacités physiques, psychologiques et sociales. Aussi, des facteurs présents dans la sphère familiale y sont-ils favorables. Ces facteurs dits de protection exercent une influence positive sur la trajectoire développementale, en réduisant les risques d'apparition de comportements antisociaux et de troubles psychopathologiques. Ce sont l'implication, la participation parentale, la réceptivité des parents à l'égard des besoins de l'enfant (Sandall, Hemmeter, Smith et McLean, 2005).

Inversement, les travaux de Wasserman (2003) et ceux de Holmes (2005), font ressortir des facteurs de risques associés au milieu familial, susceptibles d'entraver la trajectoire développementale, et de ce fait de compromettre l'adaptation des individus aux conditions du milieu. A cet effet, les violences familiales, les mauvais traitements, les liens d'attachement faibles, la consommation de drogue ou/et d'alcool, certaines pratiques parentales permissives, l'incapacité à établir des limites aux enfants un milieu socioéconomique défavorisé sont autant de facteurs de risque, qui orientent les enfants vers des comportements déviants à l'adolescence et à l'âge adulte. Les conduites et comportements des parents, peuvent ainsi déstabiliser les capacités adaptatives d'un enfant et contribuer à élevé le risque d'une trajectoire de vie orientée vers la délinquance (Savoie, 2007).

Mais, au sein de ces familles dites à risque, des enfants arrivent à se développer et à s'adapter aux exigences du milieu dans lequel ils évoluent, sans toutefois manifester de troubles comportementaux ou psychiques. Ces enfants résilients montrent qu'un individu peut poursuivre son développement malgré des conditions de vie adverses, sans être affecté négativement par des pratiques parentales parfois jugées défaillantes (Cyrunlick, 2003). 
Dans cette dynamique, la résilience se résume par la faculté à surmonter les difficultés liées aux conditions de vie et de s'adapter à un milieu donné. A cet effet, le milieu scolaire peut être considéré comme un facteur de résilience (Mucchielli, 2000). Les enfants peuvent percevoir l'institution scolaire comme un environnement sécure qui leur apporte les apprentissages essentiels, pour développer leurs potentiels. Depuis plusieurs années, on observe que bien des enfants se développent grâce ou à travers l'investissement à la scolarité. L'école n'est pas associée à une séparation affective et émotionnelle entre l'enfant et son réseau familial, mais plutôt à un environnement sécure où il peut s'épanouir (Anaut, 2002). Selon les psychologues, les causes sont diverses, des carences affectives graves dans le milieu familial, des maltraitances physique ou psychologique voire des abus en passant par les trajectoires de vie délétères marquées par les guerres ou les traumatismes liés à divers handicaps.

En ce qui concerne le handicap chez l'enfant, le contexte semble particulier. Dans cette phase fondamentale de la croissance individuelle, son développement au niveau physique, psychologique, social, culturel et scolaire peut être influencé. En effet, pour qu'un enfant soit déclaré handicapé, les conditions suivantes sont essentielles : d'abord une évaluation diagnostique doit être réalisée par un personnel qualifié. Les conditions de cette évaluation servent à préciser la nature de la déficience ou du trouble. Il faut donc souligner que biologiquement, tous les handicaps ont pour origine une déficience organique. Par exemple, le handicap sensoriel provient d'une déficience liée aux organes des sens (Oléron, 1972).

Ensuite, des incapacités et des limitations doivent découler de la déficience ou du trouble, se manifestant dans les domaines de la vie de l'enfant. En argumentant nos propos par l'exemple précédent, Chaque organe des sens ayant son utilité et son mode de fonctionnement, ainsi chaque handicap sensoriel entraine, des difficultés en relation avec l'utilité et le mode de fonctionnement de l'organe déficient. Dans cette perspective, le handicap visuel et le handicap auditif offrent la possibilité de mieux comprendre les termes incapacités et limitations. Un handicap visuel entraîne des troubles de l'acuité visuelle. De cette situation résulte des incapacités au niveau des déplacements, de l'autonomie, de la protection face aux dangers (Oléron, op cit). Un handicap auditif en plus des situations décrites, a pour conséquence des difficultés de communication avec l'entourage. Ces limitations sont parfois vécues comme un traumatisme pour l'enfant. La prise de conscience de sa différence, le regard de l'autre, les échanges limités entre celui-ci et son environnement, sont des situations auxquelles l'enfant handicapé auditif est régulièrement confronté (Oléron, 1972). Le risque le plus fréquent serait donc l’isolement (Akra, 2008). 
Mais, parfois contre toute attente, ces enfants réussissent leur scolarité malgré les conditions difficiles qu’ils vivent ou ont vécu. Il est habituel et utile de se pencher sur ce qui pose problème dans le développement des enfants et leur rapport aux apprentissages. Cependant, il est aussi fondamental de tenter de comprendre les processus à l'œuvre, dans le cadre de l'institution scolaire qui semblent participer à l'épanouissement des enfants déficients auditifs. En effet, dans quelle mesure le paradigme de résilience scolaire peut participer à l'éclairage et à la compréhension de cette situation singulière (Bouteyre, 2010).

Le concept de la résilience scolaire englobe deux facteurs: les facteurs individuels et les facteurs environnementaux. Les facteurs individuels sont des caractéristiques propres à l'enfant résilient (estime de soi par exemple). Les facteurs environnementaux, probablement les plus déterminants, en l’occurrence chez les enfants handicapés (Jourdan-Ionescu, 2014), sont représentés par les membres de la famille, plus précisément les parents. Selon plusieurs auteurs dont Théis (2006) et Békaert, Masclet et Caron (2011), les interactions relationnelles entre parents et enfant jouent un rôle dans le processus de résilience scolaire, surtout chez les enfants en situation de handicap. Celles-ci sont déterminées à travers des variables provenant du milieu socio-économique des parents telles que le niveau d’études et le style éducatif.

Le lien entre le niveau d'études et la résilience scolaire a déjà été examiné par Connor et Davidson (2003) à travers ses travaux sur l'estime de soi des enfants issus d'un milieu social défavorisé. La relation entre le style éducatif et la résilience scolaire a aussi été observée par Goldstein (2013) dans l'étude sur les enfants d’immigrés israéliens en milieu défavorisé. Il semble toutefois, que ces travaux se soient intéressés aux particularités d'enfants vivant dans des milieux défavorisés. La déficience auditive et les handicaps qui en résultent, sont une réalité qui n’a pas été examinée. Or du fait de la surdité, les enfants déficients auditifs présentent un comportement résilient atypique (0léron, 1972), d'où l'importance de notre étude. Fort de ce qui précède, nous avons choisi d’analyser dans la présente recherche, la relation entre le niveau d'études, le style éducatif des parents et la résilience scolaire chez des enfants déficients auditifs de l'Ecole Ivoirienne pour les Sourds (ECIS). Par ailleurs, les résultats d'une telle investigation sur la résilience scolaire chez l'enfant déficient auditif, peuvent donner des éclairages quant au niveau de soutien à apporter aux familles d'enfants handicapés auditifs qui vivent des situations de forte vulnérabilité. De façon pratique, elle peut conduire les spécialistes du handicap auditif et les décideurs à plus d'action en faveur de la sphère familiale.

Cette recherche repose sur l'hypothèse selon laquelle il existe un lien entre le niveau d'études, le style éducatif des parents et la résilience scolaire 
chez des enfants déficients auditifs. L’opérationnalisation de cette hypothèse nous conduit à affirmer que les enfants déficients auditifs dont les parents ont un niveau d'études primaire, développent plus la résilience scolaire protection que leurs homologues dont les parents ont un niveau d'études secondaire ou supérieur. De même, les enfants déficients auditifs dont les parents utilisent le style éducatif autoritaire, optent plus pour la résilience scolaire protection que leurs homologues dont les parents utilisent respectivement les styles éducatifs démocratique et permissif. De plus, les enfants déficients auditifs dont les parents ont un niveau d'études secondaire, adoptent plus la résilience scolaire défi comparativement à leurs pairs dont les parents ont un niveau d'études primaire ou supérieur. Les enfants déficients auditifs dont les parents adoptent le style éducatif permissif, ont également plus tendance à manifester la résilience scolaire défi par rapport à leurs pairs dont les parents sont autoritaires ou démocratiques. Par ailleurs, les enfants déficients auditifs dont les parents ont un niveau d'études supérieur, tendent plus vers la résilience scolaire compensation relativement aux autres enfants dont les parents ont un niveau d'études secondaire ou supérieur. Aussi, les enfants déficients auditifs qui ont des parents démocratiques, optent-ils plus pour la résilience scolaire défi par rapport aux autres enfants dont les parents sont permissifs ou encore autoritaires.

\section{Méthodologie}

Variables de l'étude

\section{Variables indépendantes}

\section{a. Niveau d'études des parents}

Le niveau d'études des parents représente la variable indépendante impliquée dans la présente recherche. Le concept de niveau d'études renvoie aux programmes éducatifs institués par l'école. Ceux-ci, sont regroupés à l'intérieur d'un ensemble ordonné de catégories correspondant aux connaissances, savoir-faire, savoir-être et aux compétences exigées par le système éducatif. Il constitue également un indicateur pour distinguer les individus en fonction du contenu des études effectuées à des classes représentées dans nos institutions scolaires, par des niveaux d'étude différents : niveaux d'études primaire, secondaire et supérieur (Cissé, 2000).A cet effet, la variable indépendante de notre recherche se présente selon trois modalités: Les parents de niveau d'études primaire, les parents de niveau d'études secondaire, et les parents de niveau d'études supérieur.

\section{- $\quad$ Les parents de niveau d'études primaire}

Il s'agit des parents dont le niveau d'études se limite à la dernière classe du cycle primaire, c'est-à-dire le Cours Moyen deuxième année (CM2). Selon cissé (op cit) d'un point de vue psychologique, les parents d'un niveau d'études primaire sont défavorisés par un handicap 
socioculturel. L'univers linguistiquement et culturellement pauvre ainsi que le manque de stimulation intellectuelle, a une influence sur la participation parentale au suivi scolaire. Au vu de ce qui précède, on peut se demander comment les parents d'un niveau d'études primaire peuvent-ils aisément décoder la structure scolaire, lorsque ceux-ci ont quitté tôt l'école ? A cela s’ajoutent les croyances culturelles qui dépeignent les situations telles que les handicaps comme des malédictions ou des sorts (Kouamé, 2013). Les parents d'un niveau d'études primaire peuvent ne pas posséder les outils nécessaires, pour faire face à une déficience auditive chez leur enfant. Aussi adoptent-ils, certaines attitudes éducatives qui ont des conséquences sur les relations parents/ enfants (Darling et Stenberg, 1993). Ils peuvent être plus enclins à privilégier les récompenses et les punitions, les restrictions et le contrôle systématique des différents déplacements des enfants (Kellerhards et coll., 1992) afin d'inculquer par conditionnement opérant, des valeurs de respect de l'autorité, des normes liées au travail et aux comportements socialement acceptables (Skinner, 1968). Dans ce contexte peu favorable à l'autonomie, certains enfants dont les parents ont un niveau d'études primaire, ont tendance à obtenir des performances scolaires élevées et réussissent leurs études (Darling et Stenberg, op cit). On peut donc considérer que face aux conditions averses, ces enfants se réfèrent plus facilement à leurs parents et font preuve de résilience scolaire protection (Godstein, 2013).

\section{- $\quad$ Les parents d'un niveau d'études secondaire}

C'est la catégorie de personnes dont le niveau d'études se situe entre la Sixième $\left(6^{\text {ème }}\right)$ et la Terminale $\left(\mathrm{T}^{\mathrm{le}}\right)$ pour l'enseignement général, du Certificat d'Aptitude Professionnelle (CAP) au Brevet de Technicien (BT), en ce qui concerne l'enseignement technique. Ces parents par le biais de leur niveau de scolarité plus élevé, ont acquis une certaine acuité verbale. Ils s'expriment mieux, ayant même eu la possibilité d'apprendre des langues étrangères. Néanmoins, les parents ayant abandonné les études avant la fin du cycle secondaire pour plusieurs raisons (problèmes financiers, inadaptation aux programmes éducatifs, paternité ou maternité précoce) développent ce que Calin (2012) désigne sous les vocables de sentiments de rejet et d'abandon corrélés par une faible estime de soi. Cette situation influence les attitudes éducatives de cette catégorie de la population et compromet l'exercice en générale de la parentalité (Larroque, 2010). De ce fait, certains parents de niveau d'études secondaire peuvent adopter une forme d'indifférence à l'égard de l'institution scolaire et une négligence à l'endroit des enfants ou au moins de leurs apprentissages. Cyrunlick (2003) affirme que leurs enfants usent d'un nombre de stratégies d'adaptation (mécanismes de défense et méthodes de coping) qui les amènent à penser qu'ils ne peuvent pas compter sur leurs parents déjà en échec scolaire. Pour 
ces derniers, les ressources familiales semblent insuffisantes. Ils décident de faire confiance en leurs capacités individuelles à faire face aux conditions aversives (Goldstein, 2013). Ainsi selon Goldstein (op cit), les enfants des parents de niveau d'études secondaire, développent la résilience scolaire de type défi.

\section{- Les parents d'un niveau d'études supérieur}

C'est la dernière catégorie d'individus qui correspond aux personnes ayant faites des études supérieures dans les Universités ou Grandes Ecoles. Ainsi, les établissements scolaires, les enseignants, le processus et l'expérience des études, leur sont plus familiers. Les parents étant les modèles par excellence de l'apprentissage social, il est raisonnable de supposer que les enfants de ces derniers peuvent, en observant et/ou imitant leurs géniteurs, être enclins à privilégier les formations scolaires (Bandura,1986). Du fait de leur niveau de scolarité élevé, dans un contexte de handicap, ils ont les outils symboliques nécessaires pour exécuter les comportements les plus valorisés par la société (Aka, 2008). Ainsi, les individus de niveau d'études supérieur encouragent leurs enfants au respect des normes sociales et surtout, à l'autonomie et à la valorisation de soi (Bouteyre, 2010). Les enfants déficients lorsqu'ils sont encouragés et aidés de leurs parents, ont tendance à compter sur leur potentiel, leur propre capacité à faire face à l'adversité. Ils développent à cet effet, la résilience scolaire dite compensatoire.

\section{b. Style éducatif des parents}

\section{- Le style autoritaire}

Il est caractérisé par un contrôle parental élevé, une rigidité et une certaine défiance qui ont pour conséquence : un usage fréquent de la punition (physique) et une autonomie restreinte chez l'enfant. Les parents autoritaires considèrent que leurs enfants ont peu de droits mais de nombreuses responsabilités (Baumrind, 1980). Ce style éducatif inclus les moyens tels que : la récompense, la menace, les délais, l’évaluation et la surveillance. C'est une forme de conditionnement opérant, utilisé pour contrôler le comportement des enfants (Skinner, 1968). Le style autoritaire semble avoir également un effet positif sur la conduite de l'enfant concernant : l'acquisition des valeurs de travail, d'obéissance, de respect de l'autorité et la réussite scolaire (Kellerhards, 1992). Selon Goldstein (2013), les enfants dont les parents sont autoritaires, ont aussi tendance à performer sur le plan scolaire, lorsqu'ils sont soumis à des conditions de vies adverses. Ils se réfèrent à leurs parents du fait de leur autonomie restreinte et développent donc, la résilience scolaire de type protection. On peut donc penser que le style autoritaire influence la résilience scolaire chez des enfants déficients auditifs. 


\section{- $\quad$ Le style permissif}

Ce deuxième style est caractérisé par un contrôle parental faible, un niveau de souplesse et de confiance élevé (laxisme). Les parents permissifs ont des comportements non-punitifs. Ils considèrent que leurs enfants ont les mêmes droits que les adultes mais très peu de responsabilités et exercent un certain libéralisme quant aux activités scolaires des enfants (Baumrind, 1980). Ce style peut être utilisé chez les parents ayant un niveau d'étude secondaire (kellerhards et coll, 1992). Il est également marqué par un faible niveau d'obéissance, de respect de l'autorité et d'acquisition de la valeur du travail. Ici, les enfants obtiennent généralement un faible niveau de réussite scolaire et ont plus de probabilité de développer des comportements antisociaux par rapport à leurs pairs (Steinberg et Darling, 1993). Selon Goldstein (2013), le style permissif associé à un niveau d'étude moyen chez les parents influence la résilience scolaire des enfants vivant dans des conditions aversives. La forme de résilience scolaire ici développée, semble être celle caractérisée par le défi. Cela nous laisse supposer que le style permissif influence la résilience scolaire chez les enfants déficients auditifs.

\section{- $\quad$ Le style démocratique}

Dans le style démocratique, l'enfant est encouragé à obéir aux normes extérieures. Néanmoins, il est aussi encouragé à être autonome et responsable (Baumrind, 1980). Les contextes soutenant l'autonomie favorisent la réussite scolaire même dans des conditions à risque, surtout dans le cas où les enfants sont issus de milieux urbains (Goldstein, 2013). Les parents déclarant utiliser un style souple sont les plus favorisés. Ils sont moins sévères et donne un soutien scolaire équilibré. Ainsi, on peut aussi penser que le style démocratique peut influencer la résilience scolaire de type compensatoire chez les enfants.

Les styles éducatifs semblent liés aux différentes formes de résilience scolaire. En effet à chaque style, correspondent une "constellation », un " répertoire » d'attitudes et de comportements qui caractérisent les parents (Lautrey, 1980). Le style autoritaire parental favoriserait donc la résilience scolaire protection, le style démocratique quant à lui serait un terrain propice au développement de la résilience scolaire compensatoire et le style permissif serait lié à la résilience scolaire défi.

\section{Variable dépendante}

La résilience est l'aptitude que possède l'individu à s'adapter face aux situations adverses. En milieu scolaire, c'est la capacité qu'a un enfant de parvenir à répondre favorablement aux attentes du système scolaire, à réussir malgré un handicap ou un traumatisme grave, pouvant normalement entraîner une situation d'échec (Bouteyre, 2010). Il ressort d'une étude réalisée par Masten (1990) qu'il existe trois types de dimensions relatives à 
la résilience chez des individus présentant un handicap. Dans la première dimension qui est la dimension "protection », il y a interaction des facteurs de risque et de protection et c'est cette interaction qui a un effet sur l'adaptation. Par exemple, confronté à des situations adverses, un enfant peut mobiliser ses ressources personnelles mais va surtout chercher un soutien chez ses parents. Ici, le niveau d'études primaire et le style éducatif autoritaire semblent influencer cette orientation du comportement résilient chez l'enfant. La seconde dimension est la dimension « compensatoire » où la résilience de l'enfant s'explique par le fait que ses caractéristiques individuelles lui permettent de compenser les situations adverses de son environnement. Le facteur de protection annihile le facteur de risque mais n'interagit pas avec lui. A titre d'exemple, un enfant ayant un sentiment de compétence, une perception d'efficacité et de contrôle élevés pourrait résister à des attitudes et des pratiques dévalorisantes d'un adulte. Du fait de leurs caractéristiques familiales évoquées plus haut, les enfants dont les parents ont un niveau d'études supérieur et utilisent un style éducatif démocratique ont tendance à développer cette forme de résilience scolaire, dans l'adversité. Dans la troisième dimension, "défi », la présence de facteur de risque engendre un stress considéré comme positif. Ce stress suscite un processus de développement des compétences chez l'enfant qui a des effets favorables sur l'adaptation et l'auto-détermination. L'enfant considère la réussite scolaire dans des conditions difficiles comme un défi. Du fait de leurs caractéristiques familiales, les parents qui ont un niveau d'études secondaire et qui usent du style éducatif permissif paraissent amener leurs enfants à développer cette forme de résilience scolaire.

\section{Echantillon de l'étude \\ Choix des sujets}

Dans le contexte d'études portant sur le handicap chez l'enfant, la résilience prend inévitablement une orientation particulière. Elle est confrontée aux exigences propres aux domaines du développement de l'enfant handicapé, ici de l'enfant handicapé auditif. Elle pourrait s’inscrire dans une approche écologique. Le but est de mettre notamment en lumière d'une part les capacités et les incapacités propres à l'enfant, et d'autre part, les relations avec celui-ci et son environnement. En effet, certains facteurs comme la maîtrise du langage gestuel, l'autonomie, la participation en classe, le redoublement ou encore l'âge, sont susceptibles d'influencer les manifestations du comportement résilient. Dans le but de contrôler ces sources d'influences potentielles, les élèves qui font partie de l'échantillon sont choisis à partir de critères de résilience spécifiques et à l'aide d'un questionnaire construit à cet effet. 
A l'ECIS, l'apprentissage de la langue des signes commence dès le préscolaire et continue jusqu'en classe de Cours Préparatoire deuxième année (CP2). Pendant ces trois années, les enfants apprennent les bases, les pré-requis de la langue tels que l'alphabet, les chiffres, les salutations ou encore les expressions d'usage courant. Ces trois années sont non seulement nécessaires pour acquérir une certaine maitrise de la langue, mais encore pour permettre aux enfants de s’adapter à leur handicap afin de le surmonter. En effet, ce temps d'adaptation paraît fondamental pour, par la suite apprécier la résilience chez l'enfant déficient auditif (Bouteyre, 2010). A partir de ce qui précède, la maitrise du langage gestuel représente, un indice de résilience. Ainsi, les enfants du préscolaire et des cours préparatoires première et deuxième année de l'ECIS ne sont pas sélectionnés dans notre étude sur la résilience scolaire. Il reste ceux inscrits en classe de Cours Elémentaire (CE) et de Cours Moyen (CM).

Dans le système scolaire ivoirien, l'âge de la scolarisation des enfants déficients auditifs au cours préparatoire première année est fixé à six ans. Toutefois, lorsque pour des raisons d'ordre économique ou socioculturelles, certains parents n'ont pu inscrire leurs enfants à l'âge requis, ils sont autorisés à le faire dans les trois années qui suivent, c'est-à-dire, jusqu'à l'âge de neuf ans. Du fait des bouleversements psychologiques liés à l'adolescence qui sont susceptibles d'influencer la résilience scolaire, nous décidons d'exclure de notre population d'étude, tous les enfants issus de cette catégorie d'âge. En considérant le critère de l'âge de scolarisation des enfants au cours préparatoire première année, nous avons été amenés à ne retenir dans notre échantillon que des élèves de Cours Elémentaire (CE) dont l'âge se situe entre dix et douze ans.

La capacité à suivre les apprentissages scolaires et à obtenir de bonnes performances, constitue pour les psychologues en général, un indice d'adaptation au milieu scolaire. Or, la résilience à l'école, loin d'être assimilée à la réussite scolaire est un processus dont l'aboutissement est de s'adapter afin de surmonter les difficultés (Goldstein, 2013). Ainsi, nous considérons en plus de critères cités ci-dessus, la moyenne des notes de classe obtenues lors des examens. L'enfant handicapé auditif résilient a la capacité de suivre les apprentissages scolaires sans être envahi par le traumatisme lié à son handicap. Cela nécessite un fonctionnement psychologique intègre qui peut assurer l'adaptation du sujet à son environnement. Ce fonctionnement se traduit par une absence d'expression symptomatique comportementale et /ou mentale (Théis, 2006). Ainsi, afin de déterminer si les élèves choisis remplissent le critère "absence de troubles comportementaux et mentaux », nous leur administrons le test projectif du Rorschach (1921). 
Selon Calin (2012), les échecs scolaires connus par l'élève peuvent avoir des répercussions négatives sur l'image qu'il a de sa propre personne et influer sur le processus de résilience scolaire. Afin de contrôler les effets de cette variable sur nos résultats, nous avons exclu de notre échantillon les élèves ayant redoublé au moins une fois au cours de leur cursus scolaire.

Au sein de l'ECIS, tous les élèves de CE sont à l'internat et ne rentre chez eux que les week-ends et les jours de congés ou de vacances scolaires. Il n'est donc pas possible de trouver des enfants résilients qui vivent avec leurs parents en permanence. Or, le but de notre étude est d’apprécier la résilience scolaire chez les enfants déficients auditifs en fonction du niveau d’études et des styles éducatifs parentaux. Nous avons par dépit, sélectionnés les enfants qui sont rentrés à l’internat seulement à partir de Janvier 2016.

Pour Steinberg (1990), le type de famille influence considérablement le développement des individus et la stabilité comportementale. La présente étude cherche à apprécier l'action conjointe du niveau d'études et du style éducatif des parents sur la résilience scolaire chez des enfants déficients auditifs. Elle se doit donc d'examiner les enfants vivant dans des familles où les deux parents sont effectivement présents.

Dans une étude portant sur le milieu socioéconomique et la résilience scolaire (Goldstein, 2013), il est mis en évidence une différence entre les individus provenant de milieux différents (favorisé/défavorisé). Pour neutraliser l'influence de cette variable sur la résilience scolaire des sujets, nous ne considérons que les élèves issus de milieux défavorisés

Selon les travaux de Jordan-Ionescu (2014), il y a une nette différence entre filles et garçons dans la manifestation de la résilience scolaire. Afin de contrôler l'influence de la variable sexe sur nos résultats, nous avons retenu dans chaque groupe des effectifs de filles et de garçons en nombre comparables.

En définitive, l'échantillon à l'étude se compose de neuf (9) filles et de neuf (9) garçons dont l'âge varie entre dix (10) et douze (12) ans, qui proviennent de milieux sociaux défavorisés.

\section{Instruments de l'étude}

Pour le recueil des données, nous avons utilisé comme instruments, un questionnaire d'identification des sujets, le test du Rorschach, l'Inventaire de mesure des Formes de Résilience de Békaert, Masclet et Caron, 2011 (l'IFR-40) et une Echelle de mesures des Styles Educatifs Parentaux de Terrisse et Larose, 2000 (l'ESEP). Le questionnaire d'identification que nous avons construit est destiné à recueillir les informations relatives au sexe, à l'âge des sujets, au type de famille, au niveau d'études et au niveau socioéconomique des parents. Le Rorschach révèle les troubles comportementaux et mentaux sévères, nous permettant ainsi de différencier 
les individus résilients des individus non-résilients. Quant à l'IFR-40 il met en relief les formes résilience protection, défi et compensation. Enfin, l'ESEP nous permet de distinguer les styles éducatifs parentaux (autoritaire, permissif et démocratique). La grille d'entretien que nous avons conçue a pour vocation de recueillir des informations sur la perception du handicap chez les enfants. Ces informations nous ont paru utiles dans la mesure où, celles-ci pourraient contribuer à la compréhension des réponses fournies par les sujets au questionnaire de mesure des formes d’agressivité.

\section{Procédure de collecte des données}

Cette phase de notre travail a débuté d'abord par l'administration du questionnaire d'identification des sujets, puis du test du Rorschach. Pour nous assurer que chaque question aura une réponse, cette passation a été faite avec l'aide d'un interprète en langue des signes, assigné à l'étude. Ensuite, pour déterminer les formes de résilience manifestées par les par les élèves et les styles éducatifs parentaux, les participants à l'étude ont été soumis aux différents questionnaires. Enfin, en vue de recueillir leurs témoignages sur la perception du handicap, notamment le fonctionnement de la relation parentenfant, nous avons effectué des entretiens individuels.

\section{Résultats}

\section{Aspects quantitatifs}

Tableau 1 : Effectifs des sujets présentant de la résilience scolaire protection selon le niveau d’études des parents.

\begin{tabular}{|c|c|c|c|}
\cline { 2 - 4 } \multicolumn{1}{c|}{} & $\begin{array}{c}\text { Effectifs des sujets présentant } \\
\text { la résilience scolaire } \\
\text { protection }\end{array}$ & \multicolumn{1}{c}{} \\
\hline Niveaux d'études des parents & Elevé & Faible & Total \\
\hline Primaire & 6 & 1 & 7 \\
\hline Secondaire & 2 & 4 & 6 \\
\hline Supérieur & 1 & 4 & 5 \\
\hline Total & 9 & & 18 \\
\hline
\end{tabular}

Les résultats qui figurent dans le tableau 1 indiquent que l'effectif des élèves dont les parents ont un niveau d'études primaire est plus élevé (6/18) que celui des élèves dont les parents ont un niveau d'études secondaire (4/18) et supérieur (4/18). L'analyse statistique fait apparaître que le $\mathrm{F}$ calculé $(F=6,48)$ est supérieur à $F$ théorique $(F=3,44)$ lue sur la table de Fisher à $(8,8)$ ddl et 0.05 seuil de probabilité. Il y a donc une différence significative entre les fréquences des réponses des sujets des trois groupes. Cela veut dire que l'hypothèse opérationnelle 1 de l'étude est confirmée. Autrement dit, les enfants déficients auditifs dont les parents ont un niveau d'études qui se limite à l'école primaire, développent plus la résilience 
scolaire protection comparativement à leurs homologues dont les parents ont un niveau d'études secondaire ou supérieur.

Tableau 2 : Effectifs des sujets présentant de la résilience scolaire protection selon le style éducatif des parents.

\begin{tabular}{|c|c|c|c|}
\cline { 2 - 3 } \multicolumn{1}{c|}{} & $\begin{array}{c}\text { Effectifs des sujets présentant } \\
\text { la résilience scolaire } \\
\text { protection }\end{array}$ & \multicolumn{1}{c}{} \\
\hline Styles éducatifs des parents & Elevé & Faible & Total \\
\hline Autoritaire & 5 & 2 & 7 \\
\hline Démocratique & 3 & 3 & 6 \\
\hline Permissif & 1 & 4 & 5 \\
\hline Total & 9 & 9 & 18 \\
\hline
\end{tabular}

Les résultats qui figurent dans le tableau 2 montrent que l'effectif des élèves dont les parents sont autoritaires est plus élevé (5/18) que celui des élèves dont les parents sont démocratiques (3/18) et permissifs $(1 / 18)$. L'analyse statistique fait apparaître que le $\mathrm{F}$ calculé $(\mathrm{F}=8,17)$ est supérieur à $\mathrm{F}$ théorique $(\mathrm{F}=3,44)$ lue sur la table de Fisher à $(8 ; 8)$ ddl et 0.05 seuil de probabilité. Il y a donc une différence significative entre les fréquences des réponses des sujets. L’hypothèse opérationnelle 2 est aussi confirmée. Autrement dit, les enfants déficients auditifs dont les parents sont autoritaires, développent plus la résilience scolaire protection comparativement à leurs homologues dont les parents sont démocratiques ou permissifs.

$\underline{\text { Tableau } 3}$ : Effectifs des sujets présentant de la résilience scolaire défi selon le niveau d'études des parents.

\begin{tabular}{|c|c|c|c|}
\cline { 2 - 3 } \multicolumn{1}{c|}{} & \multicolumn{2}{c|}{$\begin{array}{c}\text { Effectifs des sujets présentant } \\
\text { la résilience scolaire défi }\end{array}$} & Total \\
\hline Niveaux d'études des parents & Elevé & Faible & 7 \\
\hline Primaire & 4 & 3 & 6 \\
\hline Secondaire & 5 & 1 & 5 \\
\hline Supérieur & 2 & 3 & 18 \\
\hline Total & 11 & & 7 \\
\hline
\end{tabular}

Les résultats présentés dans le tableau 3 révèlent que l'effectif des élèves dont les parents ont un niveau d'études secondaire est plus élevé (5/18) que celui des élèves dont les parents ont un niveau d'études primaire (4/18) et supérieur (2/18). L'analyse statistique fait apparaître que le $\mathrm{F}$ calculé $(\mathrm{F}=2,04)$ est supérieur à $\mathrm{F}$ théorique $(\mathrm{F}=7,87)$ lue sur la table de Fisher à $(10 ; 6)$ ddl et 0.05 seuil de probabilité. Il y a donc aucune différence significative entre les fréquences des réponses des trois groupes. Cela veut dire que l'hypothèse opérationnelle 3 n'est pas confirmée. Autrement dit, les enfants déficients auditifs dont les parents ont un niveau d'études secondaire, 
n’adoptent pas plus la résilience scolaire défi comparativement à leurs pairs dont les parents ont un niveau d'études primaire ou supérieur.

Tableau 4 : Effectifs des sujets présentant de la résilience scolaire défi selon le style éducatif des parents.

\begin{tabular}{|c|c|c|c|}
\cline { 2 - 4 } \multicolumn{1}{c|}{} & \multicolumn{2}{c|}{$\begin{array}{c}\text { Effectifs des sujets présentant } \\
\text { la résilience scolaire défi }\end{array}$} & \multicolumn{1}{c}{ Total } \\
\hline Styles éducatifs des parents & Elevé & Faible & 7 \\
\hline Autoritaire & 3 & 3 & 6 \\
\hline Démocratique & 4 & 2 & 5 \\
\hline Permissif & 4 & 1 & 18 \\
\hline Total & 11 & 7 & \\
\hline
\end{tabular}

Les résultats qui figurent dans le tableau 4 montrent que l'effectif des élèves dont les parents sont permissif est plus élevé (4/18) que celui des élèves dont les parents sont autoritaires (3/18), mais le nombre d'élèves est le même s'agissant d'enfants dont les parents sont démocratiques et permissifs (4/18). L'analyse statistique fait apparaître que le $\mathrm{F}$ calculé ( $\mathrm{F}=$ $10,28)$ est supérieur à $F$ théorique $(F=7,87)$ lue sur la table de Fisher à $(10$; 6) ddl et 0.05 seuil de probabilité. Il y a donc une différence significative entre les fréquences des réponses des sujets. L’hypothèse opérationnelle 4 est aussi confirmée. Autrement dit, les enfants déficients auditifs dont les parents sont permissifs, développent plus la résilience scolaire défi que leurs homologues dont les parents sont autoritaires ou démocratiques.

Tableau 5 : Effectifs des sujets présentant de la résilience scolaire compensation selon le niveau d'études des parents.

\begin{tabular}{|c|c|c|c|}
\hline & $\begin{array}{r}\text { Effectifs } \\
\text { la ré } \\
\end{array}$ & $\begin{array}{l}\text { s présentant } \\
\text { scolaire } \\
\text { tion }\end{array}$ & \\
\hline Niveaux d'études des parents & Elevé & Faible & Total \\
\hline Primaire & 2 & 5 & 7 \\
\hline Secondaire & 2 & 4 & 6 \\
\hline Supérieur & 4 & 1 & 5 \\
\hline Total & 8 & 10 & 18 \\
\hline
\end{tabular}

Les résultats qui sont présentés dans le tableau 5 indiquent que l'effectif des élèves dont les parents ont un niveau d'études supérieur est plus élevé (4/18) que celui des élèves dont les parents ont un niveau d'études primaire (2/18) et secondaire (2/18). L'analyse statistique fait apparaître que le $F$ calculé $(F=5,11)$ est supérieur à $F$ théorique $(F=3,68)$ lue sur la table de Fisher à $(9 ; 7)$ ddl et 0.05 seuil de probabilité. Il y a donc une différence significative entre les fréquences des réponses des sujets des trois groupes. Cela veut dire que l'hypothèse opérationnelle 5 de l'étude est confirmée. Autrement dit, les enfants déficients auditifs dont les parents ont un niveau 
d’études supérieur, manifestent plus la résilience scolaire compensation par rapport à leurs pairs dont les parents ont un niveau d'études primaire ou secondaire.

Tableau 6 : Effectifs des sujets présentant de la résilience scolaire compensation selon le style éducatif des parents.

\begin{tabular}{|c|c|c|c|}
\cline { 2 - 3 } \multicolumn{1}{c|}{} & $\begin{array}{c}\text { Effectifs des sujets présentant } \\
\text { la résilience scolaire } \\
\text { compensation }\end{array}$ & \multicolumn{1}{c}{} \\
\hline Styles éducatifs des parents & Elevé & Faible & Total \\
\hline Autoritaire & 1 & 6 & 7 \\
\hline Démocratique & 5 & 1 & 6 \\
\hline Permissif & 2 & 3 & 5 \\
\hline Total & 8 & 10 & 18 \\
\hline
\end{tabular}

Les résultats montrent que l'effectif des élèves dont les parents sont démocratiques est plus élevé (5/18) que celui des élèves dont les parents sont autoritaires (1/18) et permissifs (2/18). L'analyse statistique fait apparaître que le $F$ calculé $(F=4,54)$ est supérieur à $F$ théorique $(F=3,68)$ lue sur la table de Fisher à $(9 ; 7)$ ddl et 0.05 seuil de probabilité. Il y a donc une différence significative entre les fréquences des réponses des sujets. L’hypothèse opérationnelle 6 est aussi confirmée. Autrement dit, les enfants déficients auditifs dont les parents sont démocratiques, adoptent plus la résilience scolaire compensation par rapport à leurs homologues dont les parents sont autoritaires ou permissifs.

\section{Analyse qualitative}

A la lumière des entrevues et des tests réalisés avec les couples de parents de niveau d'études primaire, certaines analyses peuvent être faites. En premier lieu, les parents qui se sont limités au niveau d'études primaire semblent moins outillés pour motiver leurs enfants à poursuivre leurs études. Le père de K... par exemple, mentionne : "C'est difficile quand on n'est pas allé loin à l'école d'aider son enfant à faire ses exercices». Quand à sa mère, elle affirme qu'elle-même n'ayant pas pu poursuivre ses études audelà du Cours Moyen première année, elle ne peut obliger sa fille à aller à l'école surtout qu'elle est handicapée. Elle préfère plutôt lui apprendre ce qu'elle sait faire, c'est-à-dire : le commerce de vêtements. Aussi, du fait du handicap auditif les parents d'un niveau d'études primaire adoptent-ils le style éducatif autoritaire qui laisse peu de place à l'autonomie, l'enfant n'agit pas directement sur sa vie, les différents aspects de celle-ci sont plus ou moins contrôlés par ses parents, y compris dans les activités scolaires. Ce mode de fonctionnement familial conduit les enfants à se référer à leurs parents dans les prises de décisions. Par conséquent, ils développent plus la résilience scolaire protection par rapport aux enfants déficients auditifs dont 
les parents sont d'un niveau d'études secondaire ou supérieur et ont également un niveau d'autodétermination faible relativement à leurs pairs.

En second lieu, le niveau d'études secondaire n’influence pas la résilience scolaire défi. Autrement dit, quel que soit le niveau d'études des parents, un enfant peut considérer la résilience scolaire comme un défi. La résilience scolaire de type défi, est la forme de résilience dans laquelle, l'enfant utilise ses ressources personnelles pour surmonter son handicap et s'adapter. Elle est plus fréquente selon la littérature, chez les enfants qui perçoivent l'environnement familial comme défaillant. Cela laisse supposer que quel que soit le niveau d'études des parents, l'environnement familial peut être perçu par l'enfant, comme défavorable par rapport à la réussite scolaire. Les croyances des parents face au handicap peuvent être considérer comme un élément explicatif. En effet, plusieurs parents (environ 20\% des parents interrogés), croient que les évènements inattendus qui arrivent à leur famille comme, la surdité de l'enfant, sont du fait de l'action de puissances extérieures, invisibles mais réelles. Que ce soit des «divinités » envers lesquelles on ne s'est pas acquitté de ses devoirs, les morts ou encore certains voisins malveillants. Cette croyance se retrouve également chez les parents qui ont un niveau d'études supérieur. Ces convictions culturelles semblent également influencer les attitudes des parents envers les enfants.

En effet, On remarque à travers les entretiens que, certains parents sont permissifs. Ils déclarent que, du fait du handicap de leurs enfants, ils ont du mal à assurer le rôle de parents qui consiste aussi à établir des règles précises et à punir de façon adaptée les écarts. Ainsi, les enfants sont parfois traités différemment par rapport aux autres membres de la fratrie (traitement de faveur voire indifférence). La perception du handicap vient donc influencer les conduites parentales. L'un des pères affirment par ailleurs: «S... est un enfant qui, depuis le bas âge, est difficile à comprendre. Je tolère tout ce qu'il fait, je ne le puni pas parce que la vie l'a assez puni comme ça!». Dans cet environnement permissif, des enfants déficients auditifs considèrent la réussite scolaire comme un défi.

En dernier lieu, plusieurs situations décrites révèlent que les parents de niveau d'études supérieur ont des attitudes différentes de celles des parents des groupes précédents. Ils sont engagés, ils se préoccupent des besoins et intérêts de l'enfant en accordant plus de place aux choix, aux décisions des enfants. Ils leur apportent soutien et utilisent des stratégies qui permettent d'éviter la confrontation directe, tout en établissant des règles et des limites aux enfants. Tous les parents interrogés qui ont un niveau d’études supérieur, relatent des situations où ils ont accompagné, soutenu, renforcé l'enfant dans ce qu'il fait. Ces parents peuvent également se révéler une source de motivation pour leurs enfants, dans la poursuite des études malgré le handicap auditif. Ils ont tendance à partager leur quête 
intellectuelle avec eux et à leur transmettre des aptitudes propice à la réussite. Les parents de niveau d'études supérieures, participent plus aux activités scolaires de leurs enfants et ont des attentes plus élevées quant à la réussite scolaire. L’environnement familial chaleureux contribue également à l'épanouissement des enfants handicapés de ce groupe. Le fait d'être encouragés et aidés dans l'accomplissement des tâches scolaires, a un effet sur la résilience scolaire. Ici, le niveau d’études supérieur et le style éducatif démocratique, influence la résilience scolaire de type compensation.

Ces différents témoignages des sujets nous autorisent à penser que les pratiques éducatives parentales des deux catégories de parents, présentent des différences. Il n’est donc pas étonnant que les comportements de leurs progénitures diffèrent en ce qui concerne les manifestations de la résilience scolaire (protection, défi et compensation).

\section{Discussion}

Les résultats auxquels nous sommes parvenus révèlent que le niveau d'études et le style éducatif des parents, influencent les formes de résilience scolaire développées par des enfants déficients auditifs. A l'exception du niveau d'études secondaire, qui n’influe pas sur la résilience scolaire de type défi.

Par ailleurs, les résultats des entretiens que nous avons effectués auprès des sujets, font apparaître que les différences observées, sont du fait des facteurs présents dans l'environnement familial des enfants, qui influencent la parentalité. Il semble en effet que, les enfants déficients auditifs soient accompagnés par leurs parents dans le processus de résilience scolaire. En milieu scolaire, Les parents d'un niveau d'études primaire sont parfois limités par leur niveau de scolarité, n’ayant pas pu poursuivre leurs études au-delà des classes de l'école primaire. L’institution scolaire et ses apprentissages, leur est moins familière par rapport aux parents qui ont un niveau d'études secondaire ou supérieur. L’accent est plutôt mis sur les règles et les normes qui régissent le milieu scolaire. A cet effet, ils adoptent des attitudes directives qui se traduisent par le contrôle systématique des activités des enfants, des sorties extrascolaires, des châtiments corporels en réponse à une désobéissance. Leurs enfants apprennent la résilience scolaire par le biais de récompenses et de punitions. En effet, ils affirment faire l'effort d'avoir de bonnes notes parce qu'ils sont récompensés. Et, lorsque les notes sont mauvaises, ils sont punis. Or la théorie du conditionnement opérant stipule que, un comportement à tendance à se répéter quand il est récompensé. A l'inverse, le comportement tend à disparaître quand il est puni. On peut donc dire que ces enfants déficients auditifs semblent apprendre à être résilient par conditionnement opérant. 
Les principes qui gouvernent l'éducation parentale ne se résument pas qu'en lois et en normes établies par la société. Pour faire d'un enfant, un individu résilient, c'est-à-dire un être humain capable de surmonter les conditions de vie averses afin de s’adapter au milieu dans lequel il évolue, il faudrait l'aider à développer les facultés physiques, intellectuelles, sociales et artistiques que celui-ci possède à l'état potentiel. C'est aussi, l'objectif que doit se fixer toute éducation, surtout dans un contexte de déficience chez l'enfant (Théis, 2006). Toutefois face aux handicaps, certains parents sont « désarmés ». Le manque de connaissance appropriées sur les déficiences et les limitations qu’elles génèrent, les croyances religieuses et culturelles, sont autant de facteurs défavorables à la connaissance du fonctionnement physique et psychologique de l'enfant déficient. S'agissant d'une déficience auditive, les limitations liées aux problèmes de communication, apparaissent comme un obstacle à l'adaptation de l'enfant et à celle de la famille toute entière, face au handicap. En effet, non seulement les enfants doivent passer par un processus de résilience pour surmonter le handicap et s’adapter, mais les parents peuvent être aussi amenés à devenir résilients en faisant le deuil de «l'enfant idéal ». Les parents d'un niveau d'études supérieur sont plus enclins à être des modèles par rapport aux autres parents qui ont un niveau d'études secondaire ou primaire. En fait, ils connaissent mieux le système scolaire et ses exigences, ils y sont habitués du fait d'avoir effectué des études postsecondaires. A cet effet, les enseignements et le fonctionnement de l'institution scolaire leur sont familiers, ils peuvent donc servir de modèle de réussite scolaire pour leurs enfants. On peut penser que, le modelage intervient dans ces conditions d'apprentissage par imitation. Les enfants ont appris à être résilients en observant leurs parents, comme le précise les théories de l'apprentissage par observation.

Une dernière catégorie d’enfants s’est aussi distinguée. Il s’agit d'enfants qui ont uniquement recours à leurs propres capacités, leurs ressources individuelles pour surmonter les obstacles. Ceux-là considèrent la résilience scolaire comme un défi.

Selon la théorie de Masten et coll (1990), ces enfants utilisent ce mécanisme de cognitif lorsqu'ils perçoivent leur environnement familial comme défaillant. Ils disent travailler seuls ou « $n$ 'avoir pas besoin de l'aide de papa ou maman». Ils affirment qu'ils peuvent se «débrouiller tout seul ». Lorsque leurs parents ont été interrogés sur le travail scolaire de leurs enfants, certains ont répondus préférer ne pas s'en mêler, d'autres ont dit avoir des difficultés pour communiquer avec leurs enfants, ne parlant pas eux-mêmes le langage des signes, ils se demandent même s’ils sont de «bons parents ». Ces parents perçoivent la surdité comme un «handicap social difficile voire impossible à surmonter ». L'investissement affectif sur l'enfant est gérer avec difficulté, ils deviennent permissifs (Masten et coll., 
op cit). Les résultats de l'étude montrent que les enfants de ces derniers sont plus enclins à développer la résilience scolaire défi que leurs pairs dont les parents sont autoritaires ou démocratiques. Ici, le niveau d'études n’a aucune influence sur le comportement résilient dans sa forme défi. Les attitudes et les pratiques éducatives permissives ne dépendent pas de ce facteur. La perception de la surdité de leur enfant et les croyances relatives à ce handicap semblent en être la réponse. Quelque soit le niveau d’études, la surdité est souvent perçue comme un sort ou une malédiction ou encore « une punition de la vie ». Il est possible de percevoir dans le discours des mères de ce groupe, une forme de sensibilité face à la surdité de leur enfant, sans que toutefois des comportements tendres et affectueux ne soient spécifiquement identifier. Dans cet environnement permissif où l'enfant est quelque peu abandonné, il se donne pour défi de réussir ses études.

A partir de ce qui précède, nous pouvons affirmer que les résultats de notre investigation vont dans le même sens que ceux d'Akra (2008), qui a montré qu'il existe une corrélation positive entre les performances scolaires des enfants déficients auditifs et la participation parentale. Les conclusions de notre recherche confirment dans l'ensemble, les travaux de Théis (2006). En effet, cet auteure ont fait observer que le rôle des parents est fondamentale dans le processus de résilience scolaire, à travers leur niveau de scolarité. Nos travaux vont également dans la même direction que ceux de Bouteyre (2010), ainsi que ceux de Goldstein (2013). Ces derniers révèlent que les styles éducatifs parentaux influencent les manifestations de la résilience scolaire des enfants.

\section{Conclusion}

L’école peut être une source de déception si les enfants déficients auditifs qui se sont investis dans la formation n'arrivent pas à surmonter leur handicap et à s’adapter aux exigences du milieu. Or, les participants de notre étude, parviennent à la résilience scolaire malgré les conditions de vie dans lesquelles ils évoluent. Nous voudrions ainsi, souligner l'importance du droit à l'éducation pour ces enfants handicapés. Les apprentissages scolaires doivent être adaptés à leur rythme de développement, à leurs attentes afin de faciliter l'investissement à la scolarité. Il est probable qu'en effet les croyances des parents à l'égard de la déficience auditive puissent constituer des obstacles au processus de résilience chez l'enfant. A la lumière de certains éléments recensés propres aux cultures, des programmes d'intervention socioculturelle peuvent éventuellement être suscités. Ceux-ci, doivent être vraisemblablement conçus de manière à prendre en compte l'hétérogénéité culturelle, individuelle, sociale et spirituelle ; et nécessitent donc se subordonner aux besoins particuliers de chaque communauté. Pour ce faire, le construit de résilience scolaire se doit d’être défini et 
opérationnalisé de manière à tenir compte des multiples facteurs de risque et de protection pouvant y être associé.

\section{References:}

1. Akra, F. (2008). Participation parentale et performances scolaires chez des enfants déficients auditifs. Maîtrise de Psychologie. Université de Cocody.

2. Anaut, M. (2002). Trauma, vulnérabilité et resilience en protectiion de l'enfance ? Connexions, 77(2), 101-118.

3. Anthony, E.J. (1974). A «new scientific region to explore ». The child and his family, 4, 3-15.

4. Bandura, A. (1986). L'apprentissage social. Bruxelles. Paris: Pierre Mardaga.

5. Baumrind, D. (1966). Effets of authoritative parental control of child behaviour. Child development, 35(4), 887-904.

6. Béarkert, J., Masclet, G. \& Caron, R. (2011). Validation des facteurs de résilience (IFR 40). Revue française, 57(1), 51-61.

7. Bouteyre, E. (2010). Réussite et résilience scolaire des enfants de migrants. Paris : Dunod.

8. Calin, D. (2012). Signe et sens. Quebec : PUQ.

9. Cissé, Y.J.M. (2000). Performances au basketball et niveau d'études chez les basketteurs ivoiriens. Mémoire de maîtrise de Psychologie. Université de Cocody Abidjan.

10. Connor \& Davidson (2003). Development of a new resilience scale : the connor-davidson. Depress anxiety, 18(2), 76-82.

11. Cyrunlick, B. (2003). Entrevue de chasseurs d'idées. Paris: Odile Jacob.

12. Darling, N. \& Steinberg, L. (1993). Parenting style as context: an integrative model. Psychological bulletin, 113(3), 487-496.

13. Goldstein, E. (2013). Styles éducatifs parentaux et résilience scolaire de jeunes français. Précarité et éducation familiale, 2, 307-314.

14. Holmes, J. (2005). Empowerment in consulting. Journal of Counseling and development, 69, 222-227.

15. Jourdan-Ionescu, C. (2014). Protective assessment of resilience. $2^{\mathrm{e}}$ Congrès Mondial sur la résilience. Roumanie :Timissoura.

16. Kouamé, S. (2013). Résilience scolaire des adolescents en milieu rural dans le sud-ouest ivoirien : cas de Grabo. Thèse de sociologie. Université Félix Houphouet-Boigny de Cocody.

17. Larroque, L. (2010). Influence des pratiques éducatives parentales et des pratiques pédagogiques enseignantes sur l'acquisition des normes d'internalité: approche connexionniste et expérimentale. Thèse de doctorat de psychologie. Université de Bourgogne. 
18. Lautrey, J. (1986). Classe sociale, milieu familial, intelligence. Paris : Presses Universitaires de France.

19. Masten, M. (1989). Resilience in development: implications of the study of successful adaptation for development. The emergence, 1 , 261- 294.

20. Mucchieli, L. (2000). Familles et délinquances: un bilan pluridisciplinaire de recherches francophones et anglophones. Gaëtan Morin.

21. Oléron, P. (1972). Les handicaps sensoriels .Paris: PUF.

22. Rorschach, H. (1921). The psychodiagnostic. New York: The Guilford Press.

23. Sandall, S., Hemmeter, M.L. \& Mclean, M.E. (2005). Recommandated practices: a comprehensive guide for pratical application in early intervention. Missoula early chilwood.

24. Savoie, J. (2007). La délinquance autodéclarée par les jeunes. Juristat, 27(6).

25. Sillamy, N. (2003). Dictionnaire de Psychologie. Paris: Larousse.

26. Skinner, B. F. (1969). La revolution scientifique de l'enseignement. Bruxelles: C. Dessart.

27. Terrisse, B., Larose ; F. \& Lefevre, P. (2001). La résilience : facteurs de risque et de protection dans l'environnement social et scolaire du jeune enfant. Cahiers de centre de recherche sur les formes d'éducation et d'enseignement, 14, 129-172.

28. Theis, A. (2006). Approche psychodynamique de la résilience. Thèse de Doctorat en psychologie. Université de Nancy 2.

29. Wasserman, G. (2003). Risk and protective factors of child delinquancy. Bulletin series. 